\title{
Bcl-2/IgH expression in minimal bone marrow infiltration by follicular lymphoma cells
}

\author{
YI-QUN CHE ${ }^{1,2}$, PENG LIU $^{3}$, YUE WANG ${ }^{4}$, CHANG-GONG ZHANG $^{3}$, YA-LING HAN ${ }^{4}$, \\ DI SHEN ${ }^{2}$, YING ZHANG ${ }^{4}$, CUI-LING ZHENG ${ }^{2}$, JUN QI $^{2}$ and QING-TAO WANG ${ }^{1}$ \\ ${ }^{1}$ Clinical Laboratory Department, Chao-Yang Hospital, Capital Medical University, Beijing 100020; \\ ${ }^{2}$ Clinical Laboratory Department, Cancer Hospital, Chinese Academy of Medical Sciences and \\ Peking Union Medical College, Beijing 100021; ${ }^{3}$ Department of Medical Oncology; ${ }^{4}$ National Laboratory \\ of Molecular Oncology, Cancer Institute and Hospital, Chinese Academy of Medical Sciences \\ and Peking Union Medical College, Beijing 100021, P.R. China
}

Received May 4, 2011; Accepted August 8, 2011

DOI: $10.3892 / \mathrm{mmr} .2011 .663$

\begin{abstract}
The purpose of this study was to investigate the roles of bcl-2 chromosomal translocation and $\mathrm{Bcl}-2$ protein expression in follicular lymphoma (FL) minimal bone marrow (BM) infiltration. We identified the same bcl-2/ $\mathrm{IgH}$ fusion gene in paraffin-embedded lymph node (LN) samples and BM samples using immunohistochemistry (IHC), immunocytochemistry (ICC), cytologic morphology and fluorescence in situ hybridization (FISH). The presence of the $\mathrm{Bcl}-2 / \mathrm{IgH}$ fusion gene in the $\mathrm{BM}$ samples and paraffin-embedded LN samples from 56 patients with follicular lymphomas was detected using FISH. The Bcl-2 protein levels in $\mathrm{BM}$ and paraffin-embedded tissues were quantified using ICC and IHC, respectively. Approximately $78.6 \%(44 / 56)$ of the paraffin-embedded LN tissue sections that underwent FISH analysis had a bcl-2/IgH translocation. The primary lesion was also positive for the bcl-2/IgH fusion gene, as were the BM minimal infiltrates. The bcl-2/ $\mathrm{IgH}$ rearrangement occurred in $88.6 \%$ (39/44) of the BM specimens. The bcl-2/IgH recombination rate in stage III/IV cancers was significantly different to that observed in stage I/ II cancers $(\mathrm{p}=0.041)$. In $59 \%(23 / 39)$ of the cases with $\mathrm{t}(14 ; 18)$, $\mathrm{Bcl}-2$ was found to be present as assessed by ICC. Positive $\mathrm{Bcl}-2$ ICC staining and the $\mathrm{t}(14 ; 18)$ translocation (as detected using FISH) were positively correlated $(\mathrm{p}=0.028)$. We then applied the FISH method to slides that had previously been morphologically evaluated using Wright-Giemsa staining; any slides with at least one abnormal cell were subjected to
\end{abstract}

Correspondence to: Professor Qing-Tao Wang, Clinical Laboratory Department, Chao-Yang Hospital, Capital Medical University, Beijing 100020, P.R. China

E-mail:wqt36@163.com

Key words: follicular lymphoma, fluorescence in situ hybridization, $\mathrm{Bcl}-2 / \mathrm{IgH}$, bone marrow, infiltration
FISH analysis following staining. The assessment of bcl-2/ IgH translocation status may contribute to the better detection of minimal BM infiltration by FL cells. Utilizing FISH and cytologic morphology techniques allows for earlier and more accessible BM examination.

\section{Introduction}

Due to characteristics of the bone marrow (BM) and hematopoietic cell histology, normal histopathological and immunohistochemical staining techniques used to assess non-Hodgkin's lymphoma (NHL) according to stage and level of BM invasion do not meet diagnostic needs, as microinvasive lesions are a major source of recurrent lymphoma. A total of $25-35 \%$ of NHL stage II/III lesions should more correctly be characterized as NHL stage IV.

Currently, lymphoma BM invasion is assessed with a BM smear morphologic evaluation when a variation in lymphoma cell morphology does not allow sufficient identification. The WHO requires that when $\geq 5 \%$ of the cells are naive lymphocytes, the classification is BM infiltration, whereas normal BM is characterized by $\leq 1 \%$ naive lymphocytes. However, if the BM naive lymphocyte population is $1-5 \%$, the question raised involves determining which state is normal and which state is invasive. Thus, a BM morphology evaluation, although significant, requires auxiliary means, preferably ones that reduce human error to allow for standardization. Individual treatment necessitates individualized lab diagnostics, followed by BM examination that results in the identification of personalized therapeutic targets. However, the lack of proper molecular markers is a major limitation of this personalized approach to therapy.

The incidence of the $\mathrm{t}(14 ; 18)(\mathrm{q} 32 ; \mathrm{q} 21)$ - immunoglobulin heavy chain/B-cell leukemia/lymphoma 2 (IgH/bcl-2) rearrangement is $70-95 \%$ in follicular lymphoma (FL) cases in Western countries. This translocation juxtaposes the immunoglobulin enhancer region $(\operatorname{IgH~E} \mu)$ at $14 \mathrm{q} 32$ with the bcl-2 oncogene at $18 \mathrm{q} 21$. Consequently, bcl-2 gene expression is dysregulated, leading to overproduction of the antiapoptotic 
protein $\mathrm{Bcl}-2$. Bcl-2 protein expression is used as a marker of the response to therapy and for assessing minimal infiltration in BM (1).

At the molecular level, breakpoints on chromosome 14 occur within the joining region genes $(\mathrm{JH})$ of the $\mathrm{IgH}$ locus. Most breakpoints on chromosome 18 are clustered within a 150-base pair (bp) segment at the 3' untranslated end of exon 3 of bcl-2, which is designated as the major breakpoint region (MBR). Additional breakpoints are described $30 \mathrm{~kb} \mathrm{3'}$ of the $\mathrm{MBR}$, in a region that is referred to as the minor cluster region (mcr). A third breakpoint cluster region exists at the $5^{\prime}$ end of the bcl-2 gene and is referred to as the $5^{\prime}$-bcl-2 or variant cluster region. Rearrangement partners involving the 5'-bcl-2 breakpoint include not only IgH but also the immunoglobulin light chain genes $\mathrm{k}$ and $\lambda$, although these latter rearrangements are uncommon. A number of additional breakpoints within the bcl-2 gene have been identified in an intermediate cluster region that is $3^{\prime}$ of the MBR and $5^{\prime}$ of the mor region (2). A number of recent reports suggest that FISH is superior to PCR for detecting $\mathrm{t}(14 ; 18)$ in paraffin-embedded lymph node (LN) samples, as it is more sensitive and equally specific (3). The purpose of this study was to identify possible correlations between the morphological features, histological grades, immunohistochemical parameters and bcl-2/IgH status in FL. With this information, the assessment of bcl-2/IgH may contribute to the detection of FL BM minimal infiltrates.

\section{Patients and methods}

Patients. We conducted a study on 56 patients with histologically verified FL at the Cancer Institute and Hospital, Chinese Academy of Medical Sciences and Peking Union Medical College (CAMS \& PUMC) between 2005 and 2010. The samples obtained were formalin-fixed, paraffin-embedded tissues and BM. Patients who had undergone staging BM biopsies were identified for the purpose of the treatment. Approval was obtained from The Chinese Academy of Medical Sciences and Peking Union Medical College Research Ethics Committee. Written consent was given by the patients for their information to be stored in the hospital database and be used for research. The patients included in the present study did not receive any related treatment prior to biopsy. Complete clinical information, BM and formalinfixed, paraffin-embedded pathologic materials were obtained from the patients. The clinical information on patients was collected from the medical records department at CAMS \& PUMC. The clinical records were reviewed for all patients with particular reference to age, site of initial involvement, stage at diagnosis, response to treatment and survival. The average age of the patient cohort was 39 years (range 5-74) (Table I). Baseline staging data from routine staging procedures [computed tomography (CT) scan, gallium/ positron emission tomography (PET) scan and histological examination of BM] was available: $25(44.6 \%), 21(37.5 \%)$ and $10(17.9 \%)$ patients were found to have stages I+II, III and IV forms of the disease, respectively. The international prognostic index (IPI) score was 0 to 2 in 41 cases, 3 to 5 in 13 cases and unknown in 2 patients. The performance status (PS) was 1 in 42 patients, 2 in 10 patients and unknown in 4 patients. The median follow-up was 32 months (range 5-54).
Table I. Clinical characteristics of follicular lymphoma patients.

\begin{tabular}{lr}
\hline Patients & No. \\
\hline Median age, years & 39 \\
$\leq 60$ & 32 \\
$>60$ & 24 \\
Gender & \\
Male & 29 \\
Female & 27 \\
Stage & \\
I or II & 25 \\
III & 21 \\
IV & 10 \\
BM involvement & \\
Yes & 26 \\
No & 28 \\
bcl-2/IgH rearrangement & 39 \\
Serum LDH & \\
Normal & 93 \\
Elevated & 4 \\
Unknown & \\
\hline
\end{tabular}

BM, bone marrow; LDH, lactate dehydrogenase.

Clinical characteristics. Patient characteristics such as age, gender, stage, serum lactate dehydrogenase (LDH) levels, BM involvement and presence of bcl-2/ $\mathrm{IgH}$ translocation in the patients are shown in Table I.

Immunohistochemistry (IHC). For IHC, 4- $\mu \mathrm{m}$ paraffin sections were deparaffinized, rehydrated and washed in phosphate-buffered saline (PBS) (pH 7.4). For the assessment of Bcl-2 expression, paraffin-embedded sections were placed in citrate buffer ( $\mathrm{pH}$ 6.0) and heated. The slides were blocked with $10 \%$ normal goat serum at $37^{\circ} \mathrm{C}$ for $30 \mathrm{~min}$ and then incubated with a mouse polyclonal antibody (diluted 1:100) against Bcl-2 (Dako Corp., Carpinteria, CA, USA) overnight at $37^{\circ} \mathrm{C}$. Following washing with PBS, the slides were incubated with biotinylated secondary antibody (diluted 1:100) for $30 \mathrm{~min}$ at $37^{\circ} \mathrm{C}$, followed by a streptavidin-peroxidase $(1: 100$ dilution) incubation at $37^{\circ} \mathrm{C}$ for $30 \mathrm{~min}$. Immunolabeling was visualized with a mixture of 3,3'-diaminobenzidine solution. Counterstaining was performed with hematoxylin.

The percentages of antigen-positive neoplastic cells were divided into the following categories: $0,<10,10-30,30-50$, $51-75$ and $>75 \%$. Only definite and unambiguous staining on unequivocal malignant cells was accepted as positive. For the positive control, sections from reactive LNs and tonsils were tested concomitantly. For the negative control, the primary antibodies were omitted. Each slide was evaluated independently by two pathologists, and the percentage of tumor cells that were positively stained was determined by visual estimation and recorded to the nearest $10 \%$. Disagreements were resolved by joint review on a multi-head microscope. Cases were considered to be positive if $\geq 30 \%$ of the tumor cells were positively stained. 
Table II. Comparison of Bcl-2 protein expression and bcl-2/IgH rearrangement status in the BM.

\begin{tabular}{|c|c|c|c|c|c|c|c|}
\hline \multirow[t]{2}{*}{ Parameter } & \multicolumn{2}{|c|}{ Bcl-2 } & \multirow[t]{2}{*}{ P-value } & \multicolumn{2}{|c|}{ bcl-2/IgH rearrangement } & \multirow[t]{2}{*}{ P-value } & \multirow{2}{*}{$\begin{array}{c}\text { Correlation } \\
\text { P-value }\end{array}$} \\
\hline & + & - & & + & - & & \\
\hline Age & & & n.s. & & & n.s. & n.s. \\
\hline$\geq 60$ & 10 & 14 & & 16 & 8 & & \\
\hline$<60$ & 13 & 19 & & 23 & 9 & & \\
\hline Gender & & & n.s. & & & n.s. & n.s. \\
\hline Male & 12 & 17 & & 19 & 10 & & \\
\hline Female & 11 & 16 & & 20 & 7 & & \\
\hline BM infiltration & & & 0.009 & & & 0.013 & 0.028 \\
\hline+ & 22 & 4 & & 28 & 1 & & \\
\hline- & 1 & 27 & & 11 & 16 & & \\
\hline Stage & 23 & 16 & $0.032^{\mathrm{b}}$ & 39 & 17 & $0.041^{\mathrm{b}}$ & 0.040 \\
\hline
\end{tabular}

BM, bone marrow; n.s., not significant ( $\mathrm{p}>0.05$ ); ${ }^{\mathrm{C}}$ correlation between positive immunocytochemical staining for bcl-2 and bcl-2/IgH rearrangement identified using FISH; ${ }^{b}$ statistically significant difference between stages III/IV and stages I+II. BM involvement was defined as $\geq 5 \%$ cytomorphologically naive lymphocytes present in the $\mathrm{BM}$ at diagnosis.

Immunocytochemistry (ICC). BM cells were fixed in methanol/acetic acid $(3: 1, \mathrm{v} / \mathrm{v})$ at $4^{\circ} \mathrm{C}$. Drops of single-cell suspensions were placed on slides. Following air-drying overnight, the slides were sequentially treated as IHC.

Fluorescence in situ hybridization (FISH) analysis. Interphase FISH analysis was performed on the BM and formalin-fixed, paraffin-embedded tissue sections at the Cancer Institute and Hospital, Chinese Academy of Medical Sciences and Peking Union Medical College (CAMS \& PUMC). For the detection of the bcl-2/IgH gene rearrangements, commercially available dual-color LSI bcl-2 and LSI IgH break-apart probes were used (Vysis, Downers Grove, IL, USA). Images were captured and archived using Cytovision software (Applied Imaging, Santa Clara, CA, USA). The LSI IGH/BCL2 Dual Color, Dual Fusion Translocation Probe is a mixture of the LSI IGH probe (which is labeled with SpectrumGreen) spanning approximately $1.5 \mathrm{Mb}$ and contains sequences that were homologous to essentially the entire IGH locus and sequences extending approximately $300 \mathrm{~kb}$ beyond the $3^{\prime}$ end of the IGH locus. The LSI BCL2 probe (which is labeled with SpectrumOrange) covers a region of approximately $750 \mathrm{~kb}$ that includes the entire bcl-2 gene and additional sequences that extended approximately $250 \mathrm{~kb}$ distal and proximal to the gene. To analyze the hybridization, $1 \mathrm{ml}$ of $\mathrm{BM}$ or a $2 \mu \mathrm{m}$ tissue section was used; in each sample, a total of 50-100 nuclei were examined per probe for the presence of the chromosomal aberration, and a sample was considered to be positive for the rearrangement if more than $15 \%$ of the nuclei exhibited a break-apart of the fusion signal. Blood samples from 31 normal individuals were collected; from these, mononuclear cells were obtained and used as normal controls. The CA46 cell line was used as a positive control for the bcl-2/ IgH rearrangement. The observed signals were considered to be significant if they were present in percentages above the cut-off levels that were previously established for our laboratory. The cut-off distances of red and green signals used to define fusion signals were $1.0 \mu \mathrm{m}$ for the bcl-2/IgH probe. The upper cut-off level in paraffin-embedded LN samples was 5\% ( 5 cells with the bcl- 2 translocation out of 100 cells).

Statistical analysis. We used the Fisher's exact test to compare the proportions of abnormal signals among the groups. $\mathrm{P}<0.05$ was considered to be statistically significant.

\section{Results}

Samples from 56 FL patients (histologically defined according to the REAL classification) were analyzed with IHC and ICC for Bcl-2 expression and FISH for the bcl-2/IgH fusion gene. Bcl-2 was expressed in the membrane and cytoplasm of tumor cells. Approximately $78.6 \%(44 / 56)$ of the paraffin-embedded tissue sections in the FISH study group presented with the bcl-2/IgH rearrangement (Fig. 1E). Bcl-2 stained positive (based on IHC) in $79.5 \%(35 / 44)$ of the $t(14 ; 18)$ cases (Fig. 1C). A significant positive correlation was found between Bcl-2-positive staining and $t(14 ; 18)$ based on the FISH results ( $\mathrm{p}=0.035)$, whereas the $\mathrm{bcl}-2 / \operatorname{IgH}$ rearrangement was not detected in the majority $(34 / 35 ; 97.1 \%)$ of normal LNs in the control group. No statistically significant differences in clinical features were found between Bcl-2-positive and Bcl-2negative cases.

BM samples from 56 patients were analyzed using FISH to evaluate bcl-2/IgH rearrangement status. The primary lesion was found to be positive for the bcl-2/IgH gene rearrangement. FISH was performed directly on the BM smears in cases of minimal infiltration. The rearrangement was found in $88.6 \%$ (39/44) of the BM specimens from patients whose paraffin-embedded LN sections were positive for bcl-2/IgH (Fig. $1 \mathrm{~F}$ and Table II). However, no bcl-2/IgH rearrangement was observed in any of the normal blood specimens (31/31) (Fig. 2a). The recombination rate in stage III/IV disease was significantly different from that in stage $\mathrm{I} / \mathrm{II}(\mathrm{p}=0.041)$, and no significant correlation was found between bcl-2/IgH rearrangement status and age, gender or IPI. In 59\% (23/39) of the cases with $\mathrm{t}(14 ; 18)$, the immunocytochemical staining for Bcl-2 in the BM was positive. In FL, the 

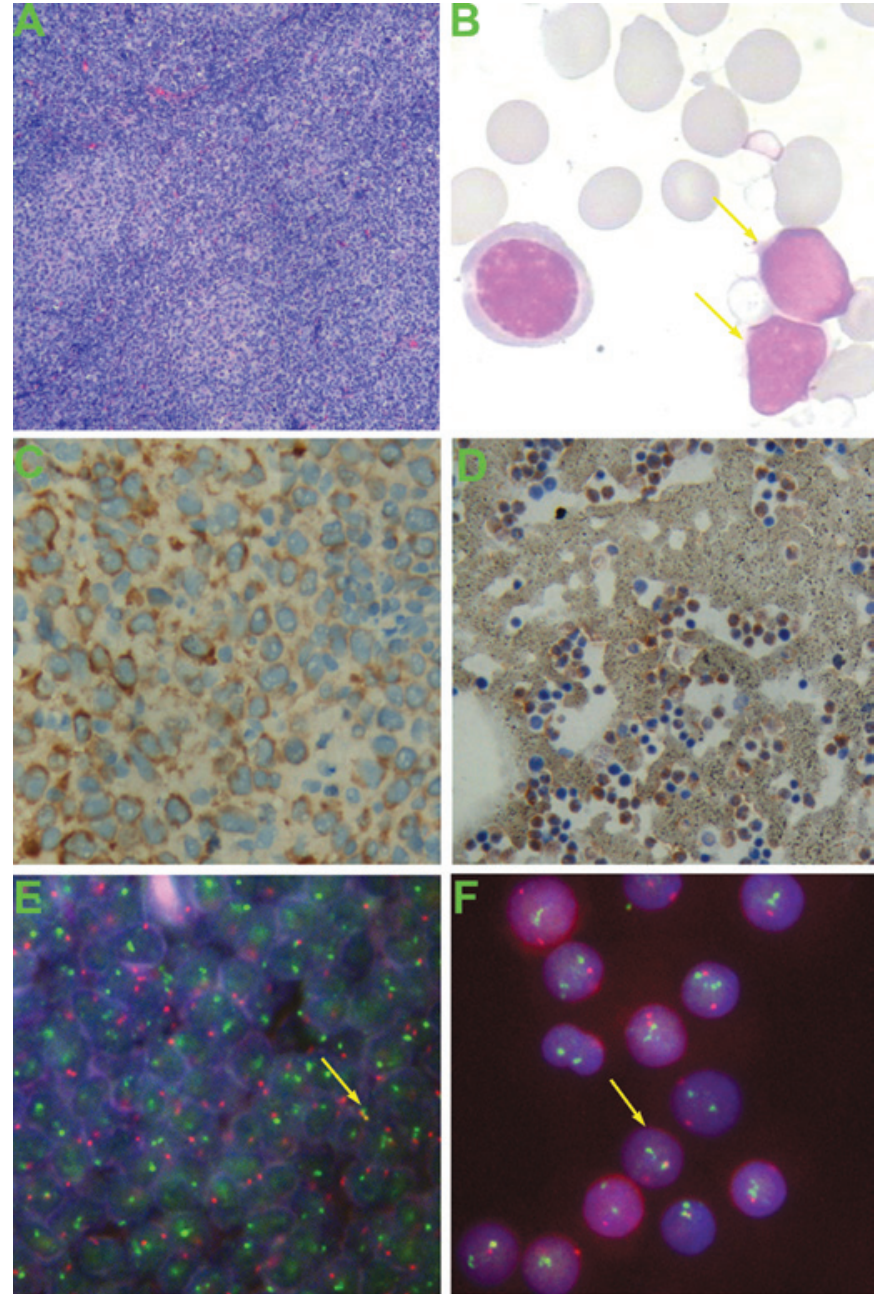

Figure 1. $\mathrm{LN}$ and $\mathrm{BM}$ sections showing bcl-2/IgH fusion products resulting from FL. (A) Analysis of a paraffin-embedded LN confirmed the presence of FL (H\&E, magnification, x100). (B) Cytomorphological assessment showed that the percentage of naive lymphocytes was $7.5 \%$; according to the FAB diagnostic criteria, this indicates BM involvement (magnification, $\mathrm{x} 1000$ ). (C) Positive IHC staining for Bcl-2 in FL tumor cells (magnification, $\mathrm{x} 400$ ). (F) Confirmation of bcl-2/IgH rearrangement using FISH (magnification, $\mathrm{x} 630$ ) and positive ICC staining for Bcl-2 in FL bone marrow. (D) Bcl-2 was expressed in the membrane and cytoplasm of tumor cells (magnification, x400). (E) Paraffin-embedded LN section $(2 \mu \mathrm{m})$ showing bcl-2/IgH rearrangement based on FISH. The FISH pattern of nuclei with $t(14 ; 18)$ translocations exhibiting fusion signals is marked by yellow staining (magnification, x630).

protein expression of Bcl-2 (derived from ICC) and the bcl-2/ IgH rearrangement were significantly correlated $(\mathrm{p}=0.028)$.

Notably, BM samples were regularly collected for morphological evaluation; any slides containing any abnormal cells were analyzed with Wright-Giemsa staining followed by FISH for the bcl-2/IgH translocation (Fig. 2C and D). Unlike standard diagnostic methods, this process identified bcl-2/IgH-positive cells with normal morphologies (11 bcl-2/ IgH-positive samples/39 samples with abnormal cells).

\section{Discussion}

Our study had two purposes: first, we used fresh BM and peripheral blood (PB) to develop a FISH assay to detect fusion
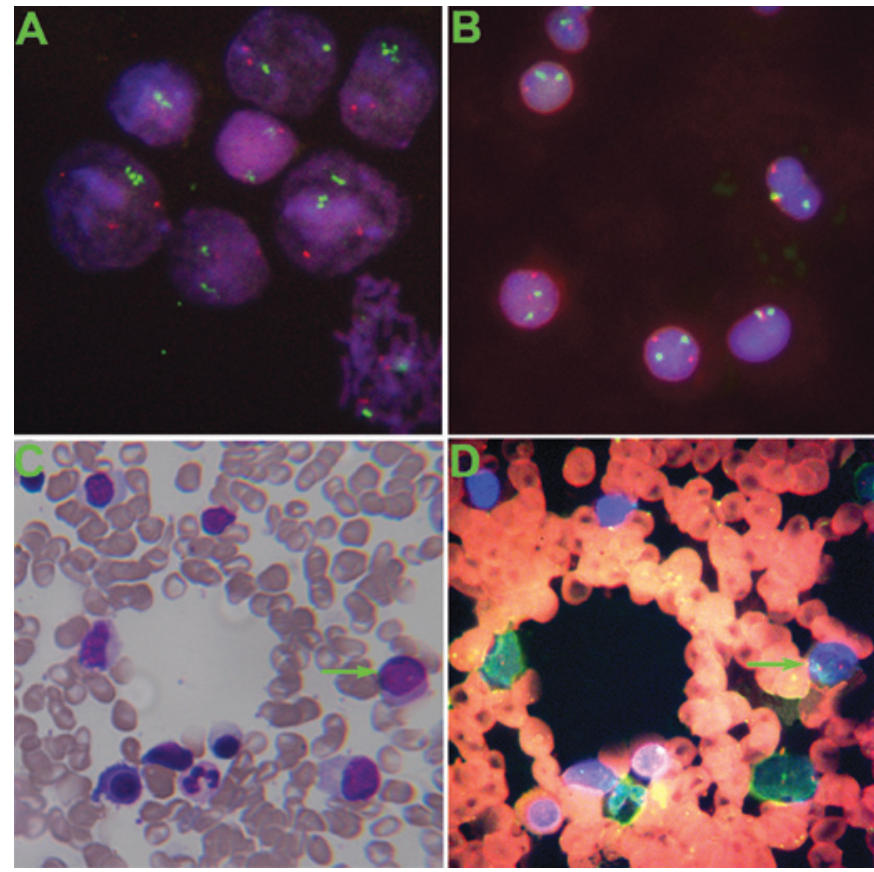

Figure 2. BM minimal infiltration morphology in combination with FISH results. (A) Negative control (magnification, x630). (B) Positive control (magnification, x630). The BM samples were regularly collected for morphological evaluation. When any abnormal cell was found on a slide, that slide was subjected to (C) Wright-Giemsa staining (magnification, x630) and then to (D) bcl-2/IgH fusion gene detection using FISH (magnification, x630).

genes in patients with the $\mathrm{t}(14 ; 18)$ translocation; second, the $\mathrm{BM}$ samples were regularly tested with morphological evaluation followed by bcl-2/IgH fusion gene detection using FISH for any slides containing abnormal cells.

Recurrent, structural cytogenetic abnormalities, most of which are balanced translocations, play a significant diagnostic and prognostic role in hematological malignancies (4). The $t(14 ; 18)$ translocation, which leads to overproduction of the Bcl-2 protein, is thought to occur in almost all FLs and is capable of being detected using FISH. Detection of this translocation is useful for monitoring the response to therapy and for assessing minimal residual disease present in the $\mathrm{BM}$ (5). However, the post-induction bcl-2/IgH status is not useful when deciding on subsequent therapy for patients with relapsed/resistant FL (6). The results show that in 59\% (23/39) of $\mathrm{t}(14 ; 18)$ cases, Bcl-2 protein was present in the BM based on ICC. In FL, Bcl-2 protein expression (as assessed with ICC) and the presence of the bcl-2/IgH rearrangement were significantly correlated. The expression level of the bcl-2/IgH fusion gene in the newly diagnosed and relapsed groups was much higher than that in the remission group.

BL-like tumors originated from the FL-like clones found

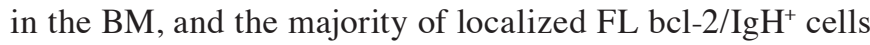
were found in the BM. Lymphoma cells may reversibly spread from the affected LN to the BM. Bcl-2/IgH expression is associated with disease status; the expression level is high in newly diagnosed and relapsed patients and low in those who have achieved remission. These results suggest that the BM is a suitable location to detect the bcl-2/IgH fusion gene. 
We found $t(14 ; 18)$ in $69.6 \%$ (39/56) of FL BM samples and in $88.6 \%$ (39/44) of the BM specimens from patients with positive staining for bcl-2/IgH in the paraffin-embedded sections. The incidence of the bcl-2/IgH gene rearrangement in Chinese FL patients was lower than that in eastern European countries, since only the bcl-2/IgH probe MBR was detectable. It has been reported that $88.5 \%$ of bcl-2/IgH rearrangements occur at the MBR breakpoint (7). The results suggest a higher rate of BM infiltration than other studies (8-10).

The number of Bcl-2 positive cells was higher in samples from patients with BM infiltration by lymphoma cells compared to samples from patients without BM involvement, which is consistent with a recent report that was based on LightCycler PCR (11). The presence of the bcl-2/IgH rearrangement in blood and $\mathrm{BM}$ is frequently detected at staging, even at FL stage I (9). Patients with histological grade 1 or 2 tumors were more likely to have the $\mathrm{t}(14 ; 18)$ translocation than not. In addition, the complete response rate was lower in the $t(14 ; 18)$-positive group compared to the $t(14 ; 18)$-negative group, and a trend toward less favorable event-free survival was observed in the $t(14 ; 18)$-positive group (12). Achieving bcl-2/IgH negativity following FL therapy results in a better prognosis (13).

Findings of studies have shown that $\mathrm{Bcl}^{-} 2^{+}$disease presented in younger patients compared to Bcl-2 disease (14), although no difference was found regarding the age at diagnosis between $\mathrm{Bcl}-2^{+}$and $\mathrm{Bcl}-2^{-}$in the present study. Although the study group included Bcl-2 protein-positive/bcl-2/ IgH-negative cases $(8.4 \%)$, the statistically significant positive correlation between the ICC expression for Bcl-2 and the $t(14 ; 18)$ translocation detected using FISH indicates that the ICC evaluation of $\mathrm{Bcl}-2$ protein expression in this study was able to reliably predict the bcl-2/IgH status. This result must be interpreted with caution due to the limited number of cases. The $\mathrm{t}(14 ; 18)$ translocation plays a significant role in the pathogenesis of FL, and its detection using FISH is a significant tool in the diagnosis, treatment and follow-up of these malignancies since the immunocytochemical testing is negative in a significant proportion of these cases.

On average, approximately $10 \mathrm{ml}$ of $\mathrm{BM}$ is required to complete the analysis of a single sample. Our assay used $1 \mathrm{ml}$ of fresh BM fluid on a single slide to perform this study. Tissue sections that were approximately $2 \mu \mathrm{m}$ thick were optimal for our analysis; increases or decreases in the thickness led to a reduced number of grid units analyzed.

In conclusion, we report a reliable and fast FISH procedure for the detection of the bcl-2/IgH translocation that may be used on slides containing abnormal cells following morphological evaluation with Wright-Giemsa staining. To the best of our knowledge, this is the first report on the use of FISH analysis of bcl-2/IgH for predicting BM minimal infiltration.

\section{Acknowledgements}

Contract grant sponsor: National Natural Science Foundation of China. Contract grant numbers: 81000977.

\section{References}

1. Gu K, Chan WC and Hawley RC: Practical detection of t $(14 ; 18)$ (IgH/BCL2) in follicular lymphoma. Arch Pathol Lab Med 132: 1355-1361, 2008.

2. Rojnuckarin P, Assanasen T, Chotipuech A, Ruangvejvorachai P Tansatit $\mathrm{M}$, Wannakrairot $\mathrm{P}$ and Intragumtornchai $\mathrm{T}$ : High frequency of BCL2 translocation in Thai patients with follicular lymphomas. Int J Hematol 86: 352-357, 2007.

3. Einerson RR, Kurtin PJ, Dayharsh GA, Kimlinger TK and Remstein ED: FISH is superior to PCR in detecting $\mathrm{t}(14 ; 18)$ (q32;q21)-IgH/bcl-2 in follicular lymphoma using paraffinembedded tissue samples. Am J Clin Pathol 124: 421-429, 2005.

4. Mitelman F, Johansson B and Mertens F: The impact of translocations and gene fusionson cancer causation. Nat Rev Cancer 7: 233-245, 2007.

5. Georgescu A, Stoicea M, Comănescu M, Dobrea C, Andrei F, Neagu M, Cionca F, Ciobanu A, Lupu A and Ardeleanu C: Prognostic and predictive significance of the bcl-2/IgH translocation in malignant follicular lymphomas. Rom J Morphol Embryol 51: 687-691, 2010.

6. van Oers MH, Tönnissen E, Van Glabbeke M, Giurgea L, Jansen JH, Klasa R, Marcus RE, Wolf M, Kimby E, Vranovsky A, Holte H, Hagenbeek A and van der Reijden BA: $\mathrm{BCL}-2 / \mathrm{IgH}$ polymerase chain reaction status at the end of induction treatment is not predictive for progression-free survival in relapsed/resistant follicular lymphoma: results of a prospective randomized EORTC 20981 phase III intergroup study. J Clin Oncol 28: 2246-2252, 2010.

7. Radojkovic M, Ristic S, Colovic M, Cemerikic-Martinovic V, Radojkovic D and Krtolica K: Molecular characteristics and prognostic significance of $\mathrm{Bcl}-2 / \mathrm{IgH}$ gene rearrangement in Serbian follicular lymphoma patients. Neoplasma 55: 421-427, 2008.

8. Pulsoni A, Starza ID, Frattarelli N, Ghia E, Carlotti E, Cavalieri E, Matturro A, Tempera $\mathrm{S}$, Rambaldi A and Foà R: Stage I/II follicular lymphoma: spread of bcl-2/IgH ${ }^{+}$cells in blood and bone marrow from primary site of disease and possibility of clearance after involved field radiotherapy. Br J Haematol 137: 216-220, 2007.

9. Arcaini L, Colombo N, Bernasconi P, Calatroni S, Passamonti F, Orlandi E, Bonfichi M, Burcheri S, Porta MD, Rumi E, Montanari F, Algarotti A, Pascutto C and Lazzarino M: Role of the molecular staging and response in the management of follicular lymphoma patients. Leuk Lymphoma 47: 1018-1022, 2006.

10. Díaz-Alderete A, Doval A, Camacho F, Verde L, Sabin P, Arranz-Sáez R, Bellas C, Corbacho C, Gil J, Perez-Martín M, Ruiz-Marcellán M, Gonzalez L, Montalbán C, Piris M and Menarguez J: Frequency of BCL2 and BCL6 translocations in follicular lymphoma: relation with histological and clinical features. Leuk Lymphoma 49: 95-101, 2008.

11. Kornacker M, Kornacker B, Schmitt C, Leo E, Ho AD and Hensel M: Commercial LightCycler-based quantitative real-time PCR compared to nested PCR for monitoring of $\mathrm{Bcl}-2 / \operatorname{IgH}$ rearrangement in patients with follicular lymphoma. Ann Hematol 88: 43-50, 2009.

12. Yanai S, Nakamura S, Takeshita M, Fujita K, Hirahashi M, Kawasaki K, Kurahara K, Sakai $\mathrm{Y}$ and Matsumoto T: Translocation $\mathrm{t}(14 ; 18) /$ IGH-BCL2 in gastrointestinal follicular lymphoma: correlation with clinicopathologic features in 48 patients. Cancer: 2010 Dec 29 (Epub ahead of print) [Pubmed: 21192062].

13. Belada D, Smolej L, Stepánková P, Beránek M, Dvoráková D, Bukac J and Malý J: Achieving Bcl-2/IgH negativity in peripheral blood/bone marrow after therapy implies better prognosis for patients with follicular lymphoma. Vnitr Lek 53: 1057-1063, 2007.

14. Montoto S, López-Guillermo A, Colomer D, Esteve J, Bosch F, Ferrer A, Villamor N, Moreno C, Campo E and Montserrat E: Incidence and clinical significance of bcl-2/IgH rearrangements in follicular lymphoma. Leuk Lymphoma 44: 71-76, 2003. 